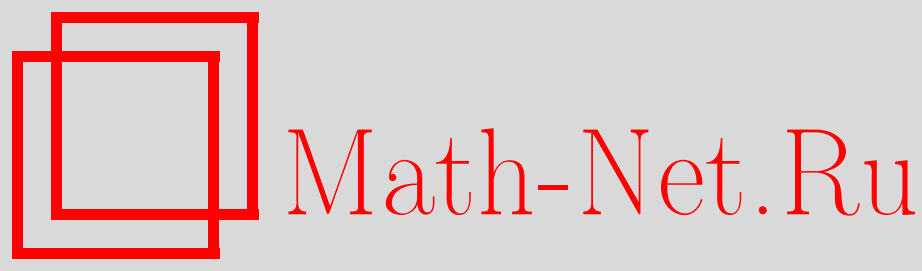

И. Я. Арефьева, М. А. Храмцов, М. Д. Тихановская, Улучшенный метод изображений для голографического описания конических дефектов, ТМФ, 2016, том 189, номер 2, 296-311

DOI: https://doi.org/10.4213/tmf9231

Использование Общероссийского математического портала Math-Net.Ru подразумевает, что вы прочитали и согласны с пользовательским соглашением http://www . mathnet.ru/rus/agreement

Параметры загрузки:

IP : 54.164 .48 .24

26 апреля 2023 г., $16: 30: 20$

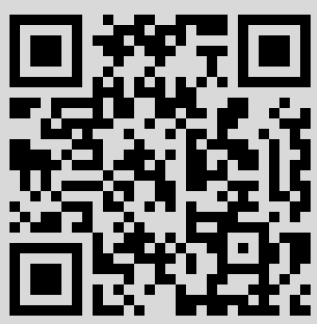




\title{
ФИЗИКА
}

Том 189, № 2

ноябрь, 2016

(C) 2016 г.

И. Я. Арефьева*, М. А. Храмцов*, М. Д. Тихановская*

\section{УЛУЧШЕННЫЙ МЕТОД ИЗОБРАЖЕНИЙ ДЛЯ ГОЛОГРАФИЧЕСКОГО ОПИСАНИЯ КОНИЧЕСКИХ ДЕФЕКТОВ}

\begin{abstract}
Геодезическое предписание в голографическом подходе в лоренцевой сигнатуре применимо только для геодезических, соединяющих пространственноподобно разделенные точки на границе, поскольку не существует времениподобных геодезических, выходящих на границу. Также, вообще говоря, не существует прямого аналитического продолжения для произвольной фоновой метрики, такой как метрика движущейся частицы в пространстве AdS. Предлагается улучшенный метод изображений для двухточечной корреляционной функции, применимый на произвольном временном интервале, когда пространство-время деформировано точечными частицами. Показано, что такое предписание находится в согласии с тем случаем, когда существует аналитическое продолжение, а также с ранее использованным предписанием для квазигеодезических. Также обсуждаются некоторые другие приложения улучшенного метода изображений: голографическая энтропия запутанности и случай многих частиц в пространстве $\mathrm{AdS}_{3}$.
\end{abstract}

Ключевые слова: AdS/CFT-соответствие, голография, геодезическое приближение, конические дефекты.

DOI: $10.4213 / \operatorname{tmf} 9231$

\section{1. ВВЕДЕНИЕ}

$\mathrm{AdS} / \mathrm{CFT-соответствие} \mathrm{и} \mathrm{голографический} \mathrm{подход} \mathrm{[1]-[4]} \mathrm{предоставляют} \mathrm{мощные}$ методы для изучения динамики сильно связанных систем как в равновесных, так и в неравновесных режимах. Некоторые из систем, допускающих голографическое описание, доступны для экспериментального изучения, например кварк-глюонная

Раздел 3 выполнен М. А. Храмцовым, остальные разделы выполнены И. Я. Арефьевой и М. Д. Тихановской, которые поддержаны грантом Российского научного фонда (проект № 14-11-00687).

* Математический институт им. В. А. Стеклова РАН, Москва, Россия. E-mail: arefeva@mi.ras.ru,khramtsov@mi.ras.ru, tikhanovskaya@mi.ras.ru 
плазма, возникающая при столкновениях тяжелых ионов [5]-[7], или квантовые жидкости и сверхпроводники [8], [9]. Это позволяет использовать голографические методы, чтобы получить некоторое понимание явлений, систематическое теоретическое описание которых отсутствует, таких как термализация и квантовые квенчи [10]-[17], явления переноса в сильно связанных системах [18], проблема квантовой запутанности [19]-[22], хаос и перемешивание в квантовой теории поля [23]. Голографическое соответствие в своем маломерном виде, так называемое $\mathrm{AdS}_{3} / \mathrm{CFT}_{2}$-соответствие, позволяет изучать трехмерную квантовую гравитацию в различных режимах. Трехмерная гравитация существенно проще гравитации в большей размерности, но она по-прежнему обладает множеством ключевых особенностей квантовой гравитации [24]. C помощью $\mathrm{AdS}_{3} / \mathrm{CFT}_{2}$-соответствия ее можно изучить, используя мощную аналитическую технику двумерной конформной теории поля [25]-[27].

Геодезическое приближение [28] играет очень важную роль в голографических вычислениях. Оно непосредственно связывает корреляционные функции конформной теории поля на границе с геометрией пространства балка. Чтобы вычислить двухточечную коррреляционную функцию между двумя точками в балке в квазиклассическом приближении, можно просуммировать по всем геодезическим в балке, соединяющим эти точки. Экстраполируя эти две точки на границу с помощью предписания Бэнкса-Дугласа-Горовица-Мартинеса (BDHM) [29], можно получить двухточечную корреляционную функцию конформной теории поля на границе. Геодезическое приближение использовалось для описания поведения таких физических величин, как вильсоновские петли в КХД во время термализации и квенча, энтропия запутанности и взаимная информация [10]-[17], [19]-[22], [30], [31]. Однако геодезическое приближение в его исходной форме [28] справедливо только для пространств с евклидовой сигнатурой или для точек, разделенных пространственноподобным интервалом, в лоренцевом случае. Времениподобные геодезические в пространствах с асимптотической AdS-метрикой не могут достигать границы, следовательно, времениподобная область недоступна для этого предписания, если, конечно, не существует аналитического продолжения исходной евклидовой формы. Из-за этого информацию, которую несет динамика при больших временах, или даже корреляторы в вещественном времени, вообе говоря, нельзя получить из геодезического приближения в произвольном пространстве-времени.

Продолжение геодезического предписания в лоренцевой сигнатуре рассматривалось ранее на некоторых пространствах с локальной AdS-метрикой. В работе [15] было построено нетривиальное евклидово продолжение для пространства-времени Вадьи. Еще один метод - это использование разрывных времениподобных геодезических, которые проходят через горизонт Пуанкаре [15], [32].

В настоящей статье мы продолжаем работу, начатую в [33], [34]. Мы предлагаем предписание для времениподобных корреляций в пространствах с локальной $\mathrm{AdS}_{3}$ метрикой, мотивированное вторым методом. Мы концентрируем свое внимание на геодезическом приближении в пространстве $\mathrm{AdS}_{3}$, деформированном точечными частицами. В недавних работах [32]-[35] для случая пространства $\mathrm{AdS}_{3}$ с точечными частицами было сформулировано геодезическое приближение двухточечной функции на границе с точками, разделенными пространственноподобным интервалом. Решения с частицами в пространстве $\mathrm{AdS}_{3}$ [36]-[39] дают конические сингулярности, вокруг которых могут наматываться геодезические. Было показано, что вклад 
в коррелятор от пространственноподобных наматывающихся геодезических, идущих с границы на границу, может быть выражен как сумма по геодезическим, которые идут до отображенных точек на границе ${ }^{1)}$. Эти изображения принадлежат орбите изометрического преобразования, определяющего топологическое отождествление (которое в случае частицы в $\mathrm{AdS}_{3}$ является отождествлением граней клина, вырезаемого частицей).

Мы используем набор вспомогательных геодезических с обращенным причинным отношением конечных точек, чтобы продолжить геодезические корреляторы за световой конус. Это также позволяет построить продолжение метода изображений, сформулированного в работе [33], на случаи, когда отождествление нарушает причинность на границе. Мы воссоздаем полюсную структуру лоренцевых корреляторов, внося соответствующие множители "руками". Мы обсуждаем обобщения рассмотренного продолжения метода изображений; мы показываем, что он может быть обобщен на случай пространства $\mathrm{AdS}_{3}$, деформированного несколькими частицами. Также мы рассматриваем приложение метода изображений для вычисления голографической энтропии запутанности в пространстве AdS с движущимся коническим дефектом.

Статья устроена следующим образом. В разделе 2 мы вводим составляющие метода изображений и преобразование отражения на границе, которое требуется для продолжения нашего предписания во времениподобную область. Мы переходим к формулировке предписания для двухточечных корреляторов на всей гранинце в разделе 3 и иллюстрируем результаты, которые дает предписание, в случае пространства $\mathrm{AdS}_{3}$ со статическим и движущимся коническими дефектами. В разделе 4 мы обсуждаем некоторые обобщения нашего метода изображений, в частности случай пространства $\mathrm{AdS}_{3}$ с несколькими частицами и предписание для голографической энтропии запутанности. Обсуждение результатов можно найти в разделе 5.

\section{2. ПРЕДВАРИТЕЛЬНЫЕ СВЕДЕНИЯ}

2.1. Пространство $\mathrm{AdS}_{3}$. Мы рассматриваем пространство $\mathrm{AdS}_{3}$ с частицей внутри. Его метрику можно получить, решая уравнение Эйнштейна в трех измерениях с отрицательной космологической постоянной и тензором энергии-импульса, соответствующим точечной частице. Частица не изменяет метрику локально и порождает коническую сингулярность [36]-[39] ${ }^{2)}$. Если частица статическая, коническая особенность располагается в центре. Следовательно, статическая частица может быть описана метрикой

$$
d s^{2}=-\operatorname{ch}^{2} \chi d t^{2}+d \chi^{2}+\operatorname{sh}^{2} \chi d \varphi^{2}, \quad \varphi \in(0,2 \pi A) .
$$

Здесь $A=1-4 G \mu \in(0,1), G$ - гравитационная постоянная, $\mu$ - масса частицы, $t-$ временная координата, $\chi$ - радиальная координата (случай $\chi \rightarrow \infty$ соответствует конформной границе). Будем обозначать угол жизненного пространства и дефицит угла как соответственно $\bar{\alpha}:=2 \pi A$ и $\alpha:=2 \pi(1-A)$.

\footnotetext{
1) Геодезическое приближение для пространства AdS с дефицитом угла сравнивалось с голографическим предписанием Губстера-Клебанова-Полякова-Виттена в работе [40].

2) Конические дефекты в контексте твердых тел в плоском трехмерном пространстве были рассмотрены в работе [41]. Голография с коническим дефектом на границе изучалась, например, в работе [42].
} 
Наиболее интересен случай частицы, движущейся внутри пространства. Поскольку частица массивная, она не может достичь границы. Можно показать, что частица будет двигаться по периодической траектории с периодом $T=2 \pi$ [28], который не зависит от массы частицы или ее быстроты $\xi$. Движущаяся частица также является глобальным дефектом в пространстве, однако соответствующее топологическое отождествление более сложное, чем поворот на постоянный угол. Как было показано в работе [33], можно получить изометрию при отождествлении координат, применяя преобразование Лоренца к граням клина, который удаляется из пространства вследствие присутствия частицы. Рассмотрим преобразование координат * :

$$
\begin{aligned}
\operatorname{tg} t^{*} & =\mathcal{B}_{\xi}(\alpha) \sec t \operatorname{th} \chi \cos \varphi+\operatorname{tg} t\left(1+2 \operatorname{sh}^{2} \xi \sin ^{2} \frac{\alpha}{2}\right) \\
\operatorname{tg} \varphi^{*} & =-\frac{2 \operatorname{tg} \varphi}{\mathcal{F}_{\xi}(\alpha)} \\
\operatorname{ch} \chi^{*} & =\operatorname{ch} \chi\left[\left(\mathcal{B}_{\xi}(\alpha) \operatorname{th} \chi \cos \varphi+\sin t\left(1+2 \operatorname{sh}^{2} \xi \sin ^{2} \frac{\alpha}{2}\right)\right)^{2}+\cos ^{2} t\right]^{1 / 2},
\end{aligned}
$$

где $\mathcal{B}_{\xi}$ и $\mathcal{F}_{\xi}$ определены как

$$
\begin{aligned}
& \mathcal{B}_{\xi}(\alpha)=\operatorname{sh} \xi\left(\sin \alpha \operatorname{tg} \varphi-2 \operatorname{ch} \xi \sin ^{2} \frac{\alpha}{2}\right), \\
& \mathcal{F}_{\xi}(\alpha)=\operatorname{ch} \xi(2 \sin \alpha \operatorname{tg} \varphi-\cos \alpha+\cos \varphi)+ \\
& +\sec \varphi \cos (\alpha+\varphi)+\cos \alpha \operatorname{ch} 2 \xi-2 \operatorname{sh}^{2} \xi .
\end{aligned}
$$

Чтобы найти изометрию на границе, которая получается при действии преобразования $*$ внутри пространства, совершим предельный переход $\chi \rightarrow \infty$ в первых двух равенствах в (2.2):

$$
\begin{aligned}
\operatorname{tg} t^{*} & =\mathcal{B}_{\xi}(\alpha) \sec t \cos \varphi+\operatorname{tg} t\left(1+2 \operatorname{sh}^{2} \xi \sin ^{2} \frac{\alpha}{2}\right), \\
\operatorname{tg} \varphi^{*} & =-\frac{2 \operatorname{tg} \varphi}{\mathcal{F}_{\xi}(\alpha)}
\end{aligned}
$$

С помощью отождествления на границе можно сформулировать предписание для отображения пространственноподобных геодезических, которое подробно объяснено в работах [32], [33]. Мы обсудим его в следующем пункте.

2.2. Намотанные геодезические и отображения. Имея изометрию *, определенную в (2.5), перейдем к описанию ее действия на всей границе. Введем обзначение $\#=*^{-1}$, а координаты граничных точек, которые получены действием изометрии $n$ раз обозначим следующим образом:

$$
(\varphi, t)^{* n}=\left(\varphi_{n}^{*}, t_{n}^{*}\right), \quad(\varphi, t)^{\# n}=\left(\varphi_{n}^{\#}, t_{n}^{\#}\right) .
$$

Между двумя данными граничными точками $a$ и $b$ может быть несколько геодезических. Геодезические, которые соединяют точки $a$ и $b$ напрямую (т. е. без пересечения клина), называются базовыми. Если существует базовая геодезическая (повторим, что только точки на границе, разделенные пространственноподобным интервалом, могут быть соединены геодезической), то она дает вклад в коррелятор, равный

$$
e^{-\Delta \mathcal{L}_{\text {ren }}(a, b)}=\left(\frac{1}{2\left(\cos \left(t_{a}-t_{b}\right)-\cos \left(\varphi_{a}-\varphi_{b}\right)\right)}\right)^{\Delta} .
$$


Данное выражение периодично по $t(t \sim t+2 \pi)$. Это означает, что вклад в коррелятор от геодезической, соединяющей точки, разделенные пространственноподобным интервалом, может быть естественно продолжен на всю временную ось с использованием условия периодичности. Для учета этого вклада мы рассматриваем световой конус, который определяется уравнением $\cos t=\cos \varphi$, а не $t=\varphi$. Здесь и далее мы рассматриваем точки $x$ и $y$, разделенные времениподобным (пространственноподобным) интервалом на границе, если $\cos \left(t_{x}-t_{y}\right)<\cos \left(\varphi_{x}-\varphi_{y}\right)$ (соответственно $\left.\cos \left(t_{x}-t_{y}\right)>\cos \left(\varphi_{x}-\varphi_{y}\right)\right)$.

Другие геодезические, которые соединяют точки $a$ и $b$, наматываются на частицу. В работах [33], [35] было показано, что перенормированные длины намотанных геодезических можно выразить через перенормированные длины отображенных геодезических ${ }^{3)}$, соединяющих точку $a$ с $b^{* n}$ и $b^{\# n}$ (или, что эквивалентно, точки $a^{* n}$ и $a^{\# n}$ с $b$ ). Следовательно, для точек, разделенных пространственноподобным интервалом, двухточечная корреляционная функция может быть записана как сумма по всем прямым геодезическим между точками внутри пространства $\mathrm{AdS}_{3}$ с дефицитом угла.

Конечно, не все отображенные точки соответствуют нужным намотанным геодезическим, поэтому в общем случае мы имеем конечное количество отображенных геодезических, которые дают вклад в коррелятор. Чтобы выделить точки, которые действительно дают вклад в сумму по всем образам, мы определяем специальные $\Theta$-функции. Их определение основано на представлении пространства $\mathrm{AdS}_{3}$ в виде цилиндра. В этом случае граничные точки принадлежат боковой границе цилиндра, и в принципе мы можем соединить любые две точки на границе прямой, проходящей внутри цилиндра. $\Theta$-функция отвечает за возможность пересечения этих прямых с клином. Функция $\Theta_{0}$ отвечает за наличие вклада от базовой геодезической, она определена следующим образом:

- $\Theta_{0}\left(\varphi_{x}, t_{x} ; \varphi_{y}, t_{y} ; \alpha, \xi\right)=1$, если прямая, соединяющая точки $\left(\varphi_{x}, t_{x}\right)$ и $\left(\varphi_{y}, t_{y}\right)$, не пересекает клин;

- $\Theta_{0}\left(\varphi_{x}, t_{x} ; \varphi_{y}, t_{y} ; \alpha, \xi\right)=0$, если прямая, соединяющая точки $\left(\varphi_{x}, t_{x}\right)$ и $\left(\varphi_{y}, t_{y}\right)$, пересекает клин.

Для определения функций $\Theta_{ \pm}$нам понадобятся некоторые вспомогательные обозначения. Обозначим грани клина как $w_{ \pm}$. При изометрии $*$, определенной в $(2.2)$, и при обратном преобразовании $\#=*^{-1}$ они преобразуются как

$$
*: w_{-} \rightarrow w_{+}, \quad \#: w_{+} \rightarrow w_{-} .
$$

Функции $\Theta_{ \pm}$определяются следующим образом:

- $\Theta_{ \pm}\left(\varphi_{x}, t_{x} ; \varphi_{y}, t_{y} ; \alpha, \xi\right)=1$, если прямая, соединяющая точки с координатами $\left(\varphi_{x}, t_{x}\right)$ и $\left(\varphi_{y}, t_{y}\right)$, сначала пересекает грань $w_{ \pm}$или если одна из граничных точек лежит на $w_{ \pm}$;

- $\Theta_{ \pm}\left(\varphi_{x}, t_{x} ; \varphi_{y}, t_{y} ; \alpha, \xi\right)=0$, если прямая, соединяющая точки с координатами $\left(\varphi_{x}, t_{x}\right)$ и $\left(\varphi_{y}, t_{y}\right)$, сначала пересекает грань $w_{\mp}$ или не пересекает граней клина.

Важно отметить, что наше определение $\Theta$-функций отличается от определения в работах [33], [34]: чтобы задать $\Theta$-функции, мы используем прямые линии вместо геодезических. Это определение может показаться противоречащим интуиции, но

3) При условии, что схема перенормировки удовлетворяет изометрии при отождествлении (см. приложение к настоящей статье и работу [33]). 


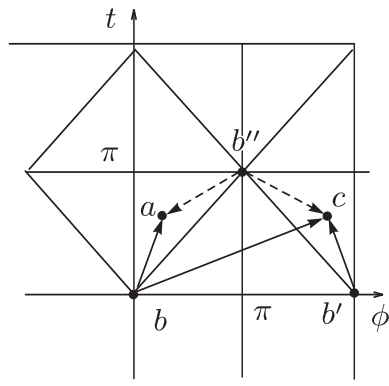

a

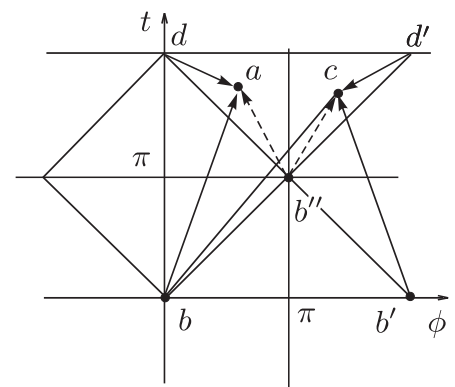

6

Рис. 1. Преобразование отражения двух векторов $\overrightarrow{b a}$ и $\overrightarrow{b^{\prime} c}$. После преобразования с учетом периодичности по угловой координате $\overrightarrow{b a} \rightarrow \overrightarrow{b^{\prime \prime}} a, \overrightarrow{b^{\prime} c} \rightarrow \overrightarrow{b^{\prime \prime} c}$ и $\overrightarrow{b c} \rightarrow b^{\prime \prime} c$; во всех случаях времениподобный интервал преобразуется в пространственноподобный (а). Преобразование отражения двух векторов $\overrightarrow{b a}$

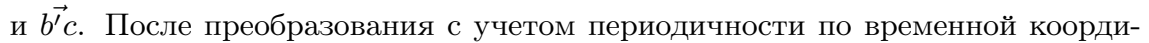
нате $\overrightarrow{b a} \rightarrow \overrightarrow{b^{\prime \prime}} a$ или $\overrightarrow{d a} \rightarrow \overrightarrow{b^{\prime \prime}} a, \overrightarrow{b^{\prime} c} \rightarrow \overrightarrow{b^{\prime \prime}} c$ или $\overrightarrow{d^{\prime}} c \rightarrow \overrightarrow{b^{\prime \prime}} c, \overrightarrow{b c} \rightarrow \overrightarrow{b^{\prime \prime}} c$ или $\overrightarrow{d^{\prime}} c \rightarrow b^{\prime \prime} c$; во всех случаях пространственноподобный интервал преобразуется во времениподобный (б).

на самом деле геодезическая, соединяющая две точки на границе, пересекает любую из граней клина тогда и только тогда, когда эти же грани пересекает прямая, соединяющая эти точки. В отличие от работ [33], [34], новые определения не связаны с существованием геодезических между граничными точками. Следовательно, мы можем использовать наше новое определение для обобщения метода на случай, когда точки разделены времениподобным интервалом.

2.3. Преобразование отражения и причинность. Если точки $x$ и $y$, лежащие на границе, разделены времениподобным интервалом, то между ними не существует непрерывной геодезической. Для обобщения нашего предписания на этот случай мы используем дополнительные пространственноподобные геодезические.

Введем полезное обозначение, чтобы построить предписание для времениподобных корреляторов. Определим отображение ${ }^{4)}$, действующее на границе пространства как

$$
R: a=(\varphi, t) \mapsto a^{R}=(\varphi+\pi, t+\pi) .
$$

Это отображение меняет причинное соотношение граничных точек при условии, что мы делаем периодическое продолжение вдоль временного направления: под действием этого преобразования времениподобный интервал $a b$ становится пространственноподобным интервалом $a b^{\prime \prime}$, и наоборот (см. рис. 1).

4)В работе [43] похожее отображение (которое называлось “диаметральным (антиподальным) отображением") было использовано, чтобы продолжить размывающие функции из патча Пуанкаре в область глобальных координат. 
Вместо геодезической между точками $x$ и $y$, разделенными времениподобным интервалом, мы можем рассмотреть пространственноподобную геодезическую, соединяющую точки $x^{R}$ и $y$. Ее вклад в коррелятор таков:

$$
\begin{aligned}
e^{-\Delta \mathcal{L}_{\text {ren }}\left(x^{R}, y\right)} & =Z\left(\frac{1}{2\left(\cos \left(t_{x}-t_{y}+\pi\right)-\cos \left(\varphi_{x}-\varphi_{y}+\pi\right)\right)}\right)^{\Delta}= \\
& =Z\left(\frac{1}{2\left(-\cos \left(t_{x}-t_{y}\right)+\cos \left(\varphi_{x}-\varphi_{y}\right)\right)}\right)^{\Delta},
\end{aligned}
$$

где $Z$ - числовой множитель, появляющийся после перенормировки (см. приложение). Заметим, что выражение в знаменателе положительно, если точки $x$ и $y$ разделены времениподобным интервалом.

Поскольку мы планируем использовать наше предписание для двухточеной корреляционной функции в координатном представлении для всей границы, мы должны задать причинную структуру лоренцевой корреляционной функции. Рассмотрим сначала коррелятор Вайтмана $\left\langle\mathcal{O}_{\Delta}\left(\varphi_{a}, t_{a}\right) \mathcal{O}_{\Delta}\left(\varphi_{b}, t_{b}\right)\right\rangle$ скалярных граничных операторов. Заметим, что коррелятор Вайтмана на цилиндре в конформной теории поля может быть записан следующим образом [44], [45]:

$$
\begin{aligned}
& \langle\mathcal{O}(t, \varphi) \mathcal{O}(0,0)\rangle=\left(\frac{1}{2(\cos (t-i \epsilon)-\cos \varphi)}\right)^{\Delta}= \\
& =\left(\frac{1}{2|\cos t-\cos \varphi|}\right)^{\Delta} e^{-i \pi \Delta \theta(-\cos t+\cos \varphi) \operatorname{sgn}(\sin t)}= \\
& =\left(\frac{1}{2|\cos t-\cos \varphi|}\right)^{\Delta} \begin{cases}e^{-i \pi \Delta \operatorname{sgn}\left(\sin t_{1}\right)}, & \text { TL-интервал, } \\
1, & \text { SL-интервал. }\end{cases}
\end{aligned}
$$

Из коррелятора Вайтмана, используя стандартные определения квантовой теории поля, можно получить причинную и запаздывающую функции Грина. Запаздывающая функция имеет вид

$$
\begin{aligned}
& G_{\text {ret }}(t, \phi)=-\frac{2 i \sin \left[\pi \Delta \operatorname{sgn}\left(\sin t_{1}\right)\right]}{\left|\cos t_{1}-\cos \phi_{1}\right|^{\Delta}} \theta\left(t_{1}\right) \theta\left(-\cos t_{1}+\cos \phi_{1}\right)= \\
& =\left(\frac{1}{2|\cos t-\cos \varphi|}\right)^{\Delta}\left\{\begin{array}{cc}
-2^{\Delta-1} i \sin [\pi \Delta \operatorname{sgn}(\sin t)] \theta(t), & \text { TL-интервал, } \\
0, & \text { SL-интервал. }
\end{array}\right.
\end{aligned}
$$

Фейнмановский пропагатор есть

$$
\begin{aligned}
& \langle T \mathcal{O}(t, \varphi) \mathcal{O}(0,0)\rangle=\left(\frac{1}{2(\cos (t-i t \epsilon)-\cos \varphi)}\right)^{\Delta}= \\
& =\left(\frac{1}{2|\cos t-\cos \varphi|}\right)^{\Delta} \begin{cases}e^{-i \pi \Delta \operatorname{sgn}\left(\sin t_{1}\right)}+\theta(-t) e^{+i \pi \Delta \operatorname{sgn}\left(\sin _{1}\right)}, & \text { TL-интервал, } \\
1, & \text { SL-интервал. }\end{cases}
\end{aligned}
$$

Здесь TL-интервал или SL-интервал означает, что соответствующее выражение имеет место для времениподобного или пространственноподобного интервала. Множитель перед фигурной скобкой каждого коррелятора равен $e^{-\Delta \mathcal{L}_{\mathrm{ren}}(t, \varphi ; 0,0)}$ для пространственноподобного случая и $e^{-\Delta \mathcal{L}_{\text {ren }}(t+\pi, \varphi+\pi ; 0,0)}$ для времениподобного случая. 
Следовательно, предписание для отображенных геодезических учитывает поведение лоренцевых корреляторов без учета причинной структуры корреляцонной функции. В окончательной формуле метода изображений необходимо будет восстановить этот множитель.

Отметим, что существует альтернативный метод отраженных геодезических квазигеодезические [15], [33], которые являются разрывными кривыми и состоят из пространственноподобных и светоподобных геодезических. Они дают тот же результат и также не несут никакой информации о причинности двухточечной функции.

\section{3. ПРЕДПИСАНИЕ ДЛЯ ДВУХТОЧЕЧНОЙ КОРРЕЛЯЦИОННОЙ ФУНКЦИИ}

3.1. Общая формула. Теперь мы имеем возможность построить двухточечную корреляционную функцию скалярных операторов с конформной размерностью $\Delta$ между точками $a$ и $b$, разделенными произвольным интервалом. В последующих формулах верхний индекс $A$ может принимать значения $\mathrm{W}$, с и ret, и тогда он обозначает корреляционную функцию Вайтмана, причинную и запаздывающую корреляционные функции. Запишем коррелятор в общем виде

$$
\begin{aligned}
G_{\Delta}^{A}\left(t_{a}, \varphi_{a} ; t_{b}, \varphi_{b}\right)= & G_{\Delta, 0}\left(\varphi_{a}, t_{a} ; \varphi_{b}, t_{b}\right) \Theta_{0}\left(\varphi_{a}, t_{a} ; \varphi_{b}, t_{b}\right)+ \\
& +\sum_{n} G_{\Delta, n}^{A}\left(\varphi_{a}, t_{a} ; \varphi_{b, n}^{*}, t_{b, n}^{*}\right) \Theta_{+}\left(\varphi_{a}, t_{a} ; \varphi_{b, n}^{*}, t_{b, n}^{*}\right)+ \\
& +\sum_{n} G_{\Delta, n}^{A}\left(\varphi_{a}, t_{a} ; \varphi_{b, n}^{\#}, t_{b, n}^{\#}\right) \Theta_{-}\left(\varphi_{a}, t_{a} ; \varphi_{b, n}^{\#}, t_{b, n}^{\#}\right),
\end{aligned}
$$

где функции $G_{\Delta, n}^{A}$ суть функции $G_{\Delta, 0}^{A}$, умноженные на перенормировочные множители (см. формулу (П.1) приложения). Функция $G_{\Delta, 0}^{A}$ определена следующим образом. Если точки $x$ и $y$ разделены пространственноподобным интервалом, то

$$
\begin{aligned}
& G_{\Delta, 0}^{\mathrm{W}}\left(\varphi_{x}, t_{x} ; \varphi_{y}, t_{y}\right)=G_{\Delta, 0}^{\mathrm{c}}\left(\varphi_{x}, t_{x} ; \varphi_{y}, t_{y}\right)=e^{-\Delta \mathcal{L}_{\mathrm{ren}}(x, y)}, \\
& G_{\Delta, 0}^{\mathrm{ret}}\left(\varphi_{x}, t_{x} ; \varphi_{y}, t_{y}\right)=0
\end{aligned}
$$

где $\mathcal{L}_{\text {ren }}(x, y)$ - перенормированная длина геодезической между точками $x$ и $y$. Схема перенормировки описана в приложении.

Если точки $x$ и $y$ разделены времениподобным интервалом, то

$$
\begin{aligned}
G_{\Delta, 0}^{\mathrm{W}}\left(\varphi_{x}, t_{x} ; \varphi_{y}, t_{y}\right)= & e^{-\Delta \mathcal{L}_{\text {ren }}\left(x^{R}, y\right)} e^{-i \pi \Delta \operatorname{sgn}\left(t_{x}-t_{y}\right)}, \\
G_{\Delta, 0}^{\mathrm{c}}\left(\varphi_{x}, t_{x} ; \varphi_{y}, t_{y}\right)= & e^{-\Delta \mathcal{L}_{\mathrm{ren}}\left(x^{R}, y\right)} \times \\
& \times\left(\theta\left(t_{x}-t_{y}\right) e^{-i \pi \Delta \operatorname{sgn}\left(\sin \left(t_{x}-t_{y}\right)\right)}+\theta\left(t_{y}-t_{x}\right) e^{+i \pi \Delta \operatorname{sgn}\left(\sin \left(t_{x}-t_{y}\right)\right)}\right), \\
G_{\Delta, 0}^{\mathrm{ret}}\left(\varphi_{x}, t_{x} ; \varphi_{y}, t_{y}\right)= & -e^{-\Delta \mathcal{L}_{\mathrm{ren}}\left(x^{R}, y\right)} \cdot 2^{\Delta-1} i \sin \left[\pi \Delta \operatorname{sgn}\left(\sin \left(t_{x}-t_{y}\right)\right)\right] \theta\left(t_{x}-t_{y}\right),
\end{aligned}
$$

где $\left(\varphi_{x}, t_{x R}\right)=\left(\varphi_{x}+\pi, t_{x}+\pi\right)$ - точка $x$, к которой применено преобразование отражения $R$, сдвигающее координаты граничной точки на $\pi$.

$\Theta$-функции, определенные в п. 2.2, обеспечивают ограничение количества членов в сумме по образам. Свойство преобразования отражения, заключающееся в том, что оно обращает причинность, гарантирует, что функция $G_{\Delta, 0}$ определена корректно на всей границе цилиндра. Заметим, однако, что изометрия (2.5) изменяет 


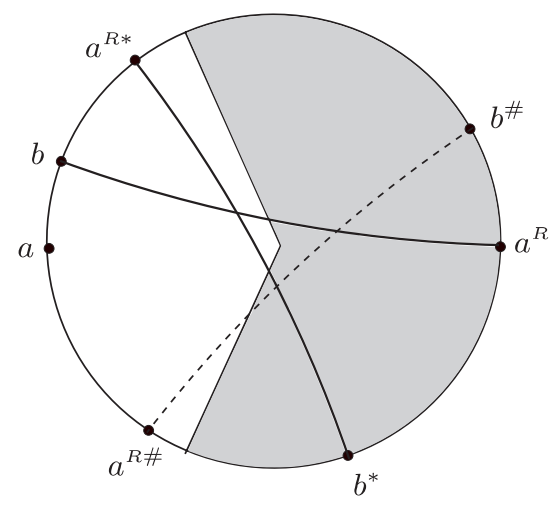

Рис. 2. Схематичный рисунок намотанной геодезической, соединяющей точки $a^{R *}$ и $b$. Длина геодезической между точками $a^{R \#}$ и $b$ не равна длине изначальной отраженной геодезической между точками $a^{R}$ и $b$. Здесь $\bar{\alpha}=5 \pi / 6$ и рассматривается случай статической частицы.

не только угловую, но и временную координату, поэтому в общем случае движущейся частицы образы $b^{* n}$ или $b^{\# n}$ могут быть отделены от точки $a$ времениподобным интервалом, даже если изначально точки $a$ и $b$ были разделены пространственноподобным интервалом. Также если точка $x$ принадлежит жизненному пространству, то точка $x^{R}$ в общем случае может быть расположена в мертвой зоне. Следовательно, отображенная геодезическая в общем случае не является базовой, а наматывается на дефект. В дальнейшем мы покажем, что можно построить намотанную геодезическую, используя тот же набор образов, что применялся для построения пространственноподобных наматывающихся геодезических.

3.2. Намотки отраженных геодезических. Заметим, что для статической частицы отождествление (2.5) приводит к отождествлению углов $\varphi \sim \varphi+\bar{\alpha}$. Полная корреляционная функция не зависит от фактического положения клина в пространстве $\mathrm{AdS}_{3}$, представленном в виде цилиндра. Следовательно, если

$$
\left|\varphi_{a^{R}}-\varphi_{b}\right|<\bar{\alpha} \quad(\bmod 2 \pi)
$$

то мы можем повернуть клин так, чтобы вся отраженная геодезическая поместилась в жизненное пространство. Если неравенство (3.2) не выполнено, то нужно строить намотку. Это же касается случая движущейся частицы при любом значении углов.

Намотки для отраженных геодезических строятся следующим образом. Пусть положение клина фиксировано. Базовая геодезическая состоит из двух частей: одна лежит в жизненном пространстве, другая - в мертвой зоне. Мы можем $n$ раз применить преобразование $*$ или \# для обеих точек и в конечном счете точка $a^{R n *}$ или $a^{R n \#}$ будет лежать в жизненном пространстве. Например, если угол жизненного пространства равен $5 \pi / 6$, то $n=1$ (см. рис. 2). Только одна геодезическая дает правильный ответ для длины базовой геодезической. На рис. 2 базовой является геодезическая между точками $a^{R *}$ и $b^{*}$.

Можно обобщить предыдущий случай на произвольное значение угла $\alpha$. Если $n \neq 1$, мы получаем геодезическую с несколькими намотками, построенную следующим 
образом. Полная геодезическая состоит из нескольких частей: геодезических между точками $a^{R}$ и $b, a^{R *}$ и $b^{*}, \ldots, a^{R n *}$ и $b^{n *}$ или $a^{R}$ и $b, a^{R \#}$ и $b^{\#}, \ldots, a^{R n \#}$ и $b^{n \#}$. Заметим также, что, поскольку мы не вводим новые граничные точки, нет необходимости вводить новые перенормировочные множители.

Чтобы учесть вклады от отраженных геодезических, к которым применены преобразования *, \#, будем действовать аналогичным способом. Во-первых, определим $\Theta$-функции и посчитаем все образы, которые дают вклад. Затем проверим интервал между каждой отображенной точкой и точкой $a$ (определим, будет он пространственноподобным или времениподобным). Пространственноподобные отображенные геодезические учитываются напрямую. Если точки разделены времениподобным интервалом, то мы применяем отражение и строим намотку для отраженной геодезической описанным выше способом для каждой отображенной точки.

\section{3. Примеры.}

3.3.1. Покоящаяся частица. Сначала рассмотрим важный частный случай. Если в случае покоящейся частицы дефицит угла составляет $2 \pi / N$, где $N \in \mathbb{Z}$, то пространство является орбифолдом $\mathrm{AdS}_{3} / \mathbb{Z}_{N}$ и сумма по образам содержит конечное число $N$ слагаемых. Следовательно, две суммы в (3.1) преобразуются в одну конечную сумму по всем $N$ образам и все оставшиеся $\Theta$-функции равны единице всюду в жизненном пространстве:

$$
\left\langle\mathcal{O}_{\Delta}(t, \vartheta) \mathcal{O}_{\Delta}(0,0)\right\rangle=\sum_{k=0}^{N-1}\left(\frac{1}{2(\cos t-\cos (\vartheta+2 \pi k / N))}\right)^{\Delta}
$$

Этот результат соответствует конформной теории поля на $\mathbb{Z}_{N}$-орбифолде, а также выражению, полученному из традиционного предписания Губстера-Клебанова-Полякова-Виттена [40].

В общем случае статической частицы имеется возможность сделать аналитическое продолжение в евклидову область и получить геодезическое предписание в полном лоренцевом пространстве, делая обратный поворот (конечно, в этом случае нужно ввести лоренцеву функцию Грина, вводя поправку $i \epsilon$ ). Наше предписание согласуется с этим продолжением. Можно обнаружить, что в случае, когда пространство не является орбифолдом, Ө-функции дают разрывы, результатом которых оказывается зонная структура корреляторов [33]. Это, по-видимому, артефакт нашего метода, поскольку мы не учитываем поправки от гравитационного взаимодействия с частицей [40]. Случаи, когда пространство не является $(\alpha=4 \pi / 3+0.2)$ и является $(\alpha=3 \pi / 2)$ орбифолдом, в сравнении показаны на рис. 3.

3.3.2. Движущаяся частица. В случае движущейся частицы мы наблюдаем (см. рис. 4), что присутствует центральная зона, которая уменьшается с уменьшением жизненного пространства. Особенности также становятся более плотными, когда жизненное пространство маленькое, что согласуется со случаем статической частицы при различных размерах клина. Зависимость корреляционной функции от времени также отражает этот факт. Графики зависимости функции $G^{-1}(t)$ от времени на интервале $t \in[-\pi, \pi]$ при фиксированных $t_{a}=0$ и $\varphi_{a}, \varphi_{b}$ для статической и движущейся частицы приведены на рис. 5. 

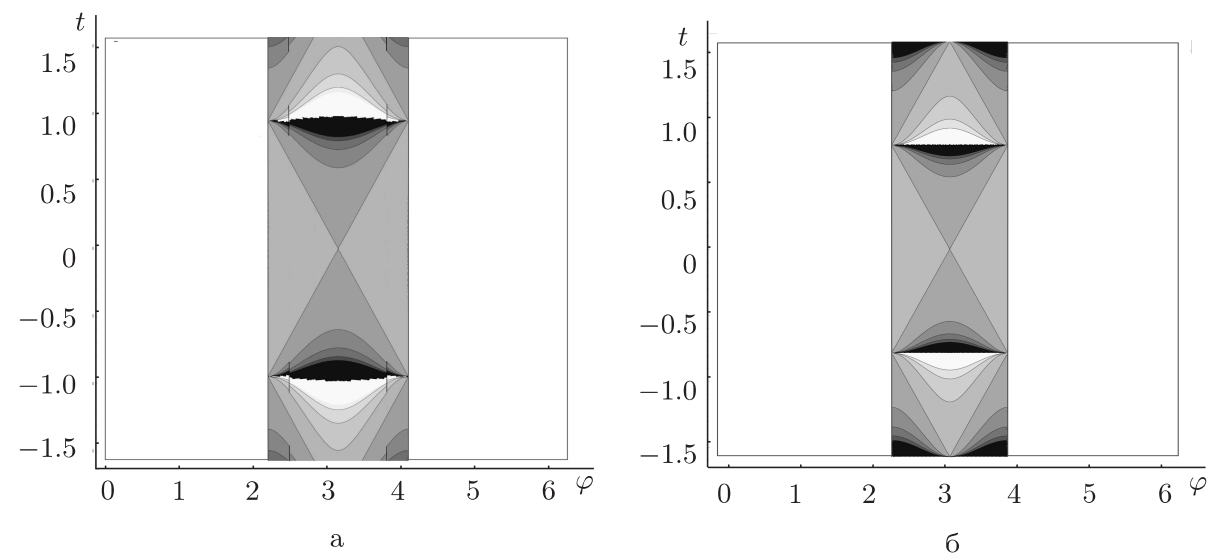

Рис. 3. График плотности функции $G^{-1}(\varphi, t)$ в случае статической частицы при $\varphi_{a}=\pi, t_{a}=0$ для $\alpha=4 \pi / 3+0.2$ (а) и $\alpha=3 \pi / 2$ (б).
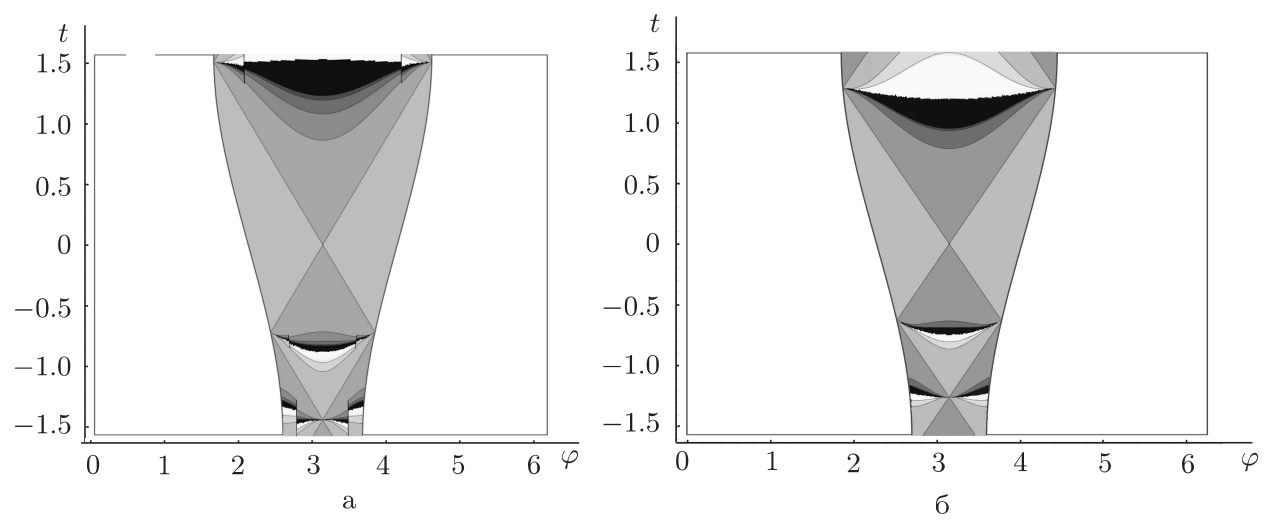

Рис. 4. График плотности функции $G^{-1}(\varphi, t)$ в случае движущейся частицы при $\varphi_{a}=\pi, t_{a}=0, \xi=0.6$ для $\alpha=4 \pi / 3+0.2$ (а) и $\alpha=3 \pi / 2$ (б).

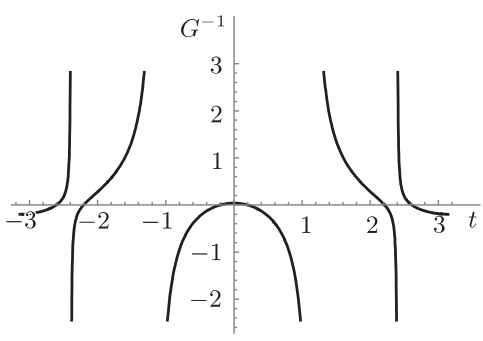

a

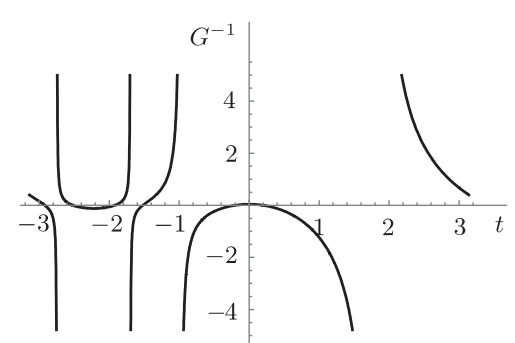

б

Рис. 5. График функции $G^{-1}(t)$ при $\varphi_{a}=\pi, t_{a}=0 ; \varphi_{b}=\pi+0.2, \bar{\alpha}=2.3 \pi / 3$ в статическом случае (а) и при $\xi=0.6$ (б). 


\section{4. ОБОБЩЕНИЯ МЕТОДА ИЗОБРАЖЕНИЙ}

4.1. Случай многих частиц. Наше предписание для вычисления коррелятора можно обобщить на случай, когда пространство в балке деформировано несколькими частицами ${ }^{5)}$. Предположим, что в пространстве имеются $P$ частиц, в результате чего возникают изометрии $*_{1}, \ldots, *_{P}$ (и аналогично для \#). Тогда метод изображений для корреляторов будет иметь вид (здесь $A=\mathrm{W}, \mathrm{c}, \mathrm{ret})$

$$
\begin{aligned}
G_{\Delta}^{A}(a, b)= & G_{\Delta, 0}^{A}(a, b) \Theta_{0}(a, b)+ \\
& +\sum_{\sigma \in S^{P}} \sum_{n_{1}, \ldots, n_{P}} \sum_{\star=*, \#} G_{\Delta, n}^{A}\left(a, b^{\left.\star_{\sigma(1)}^{n_{1}} \cdots \cdots \star_{\sigma(P)}^{n_{P}}\right)}\right) \Theta\left(a, b^{\left.\star_{\sigma(1)}^{n_{1}} \cdot \ldots \cdot \star_{\sigma(P)}^{n^{P}}\right) .}\right.
\end{aligned}
$$

В этом случае мы имеем сумму по всевозможным комбинациям всех $P$ прямых и обратных изометрий; $\Theta$-функция равна произведению $\Theta_{ \pm}^{(k)}$-функций (где индекс $k$ отвечает за перечисление всех изометрий $\star$, возникающих в функции $G$ ) и является обобщением одночастичной $\Theta$-функции, определенной для $k$-го клина так же, как и в п. 2.2. Значение индекса функции $\Theta_{ \pm}^{(k)}$ есть плюс, если индекс изометрии $\star_{k}=*_{k}$, и минус, если $\star_{k}=\#_{k}$.

4.2. Голографическая энтропия запутанности и отображения. В этом пункте мы объясняем, как можно использовать наше предписание для вычисления голографической энтропии запутанности. В частности, полезно проследить за временным поведением энтропии запутанности, когда внутри пространства находится движущаяся массивная частица. Заметим, что в случае движущихся ультрарелятивистских частиц голографическая энтропия запутанности была вычислена в работе [34].

Формула для энтропии запутанности граничной области между точками $a$ и $b$ в нашем случае имеет вид

$$
S(a, b)=\min \left\{\mathcal{L}_{\text {ren }}(a, b), \mathcal{L}_{\text {ren }}\left(a, b_{K}^{*}\right), \mathcal{L}_{\text {ren }}\left(a, b_{L}^{\#}\right)\right\}
$$

Здесь $K$ и $L$ - это минимальные целые числа, при которых выполнены условия $\Theta_{+}\left(a ; b_{K}^{*}\right)=1$ и $\Theta_{-}\left(a ; b_{L}^{\#}\right)=1$. Для вычисления энтропии необходимо выбрать наиболее короткую перенормированную геодезическую из базовой и двух намотанных геодезических. Отображенная геодезическая, которая соответствует намотке, не является одновременной в случае движущейся частицы. Для некоторых значений параметров $\xi$ и $\alpha$ интервал между точками $a$ и $b_{K}^{*}$ (или $\left.b_{L}^{\#}\right)$ может стать времениподобным, если расстояние между точками достаточно мало. Однако, несмотря на видимые сходства, этот случай несколько отличается от случая двухточечной корреляционной функции. Следует иметь в виду, что отображенные геодезические представляют собой просто удобные объекты для вычисления длин наматывающихся геодезических. Намотки, которые могут давать вклад в энтропию, всегда являются одновременными геодезическими; их необходимо учесть безотносительно к тому, движется ли частица, из-за лоренц-ковариантности в балке. Отсутствие видимого вклада от точек, разделенных времениподобным интервалом, в случае энтропии запутанности является чистым артефактом предписания, тогда как в случае корреляторов это артефакт самого́ геодезического приближения.

${ }^{5)}$ В работе [34] был рассмотрен частный случай сталкивающихся ультрарелятивистских частиц (для пространственноподобных корреляторов). 
С учетом вышесказанного теперь понятно, что нужно найти способ сохранить вклад от геодезической с намоткой при нахождении минимума для вычисления энтропии, если некоторые отображенные точки оказались во времениподобной области. Повторим, что длина геодезической между точками $x$ и $y$ в пространстве $\mathrm{AdS}_{3}$ определяется как

$$
\mathcal{L}=\ln \left(2\left(\cos \left(t_{x}-t_{y}\right)-\cos \left(\varphi_{x}-\varphi_{y}\right)\right)\right) .
$$

Если точки $x$ и $y$ разделены пространственноподобным интервалом, то выражение в скобках положительно, а если интервал времениподобный, то отрицательно; следовательно, мы получаем комплекснозначный ответ для длины геодезической. Если точки $x$ и $y$ разделены времениподобным интервалом, то точки $x$ и $y^{R}$ разделены пространственноподобным интервалом; здесь точка $y^{R}$ - это отражение точки $y$, определенное в (2.9). Таким образом, мы регулируем наше предписание так, что если точки $x, y$ разделены пространственноподобным интервалом, то соответствующий вклад в энтропию вычисляется как (перенормированная) длина геодезической $\mathcal{L}_{\text {ren }}\left(x, y^{R}\right)$. Более подходящая формула для расчета энтропии запутанности имеет вид (здесь $\left.t_{a}=t_{b}=t\right)$

$$
\begin{aligned}
S(a, b)=\min \{ & \ln \left(2\left(1-\cos \left(\varphi_{a}-\varphi_{b}\right)\right)\right), \\
& \ln \left(2\left|\cos \left(t-t^{*}\right)-\cos \left(\varphi_{a}-\varphi_{b, K}^{*}\right)\right|\right), \\
& \left.\ln \left(2\left|\cos \left(t-t^{\#}\right)-\cos \left(\varphi_{a}-\varphi_{b, L}^{\#}\right)\right|\right)\right\} .
\end{aligned}
$$

Эта формула позволяет проанализировать зависимость энтропии запутанности от времени $t$ на произвольном интервале, когда внутри пространства присутствует одна или несколько движущихся частиц ${ }^{6)}$.

\section{5. ЗАКЛЮЧЕНИЕ}

Мы предложили предписание для вычисления двухточечных корреляторов на границе и голографической энтропии запутанности, позволяющее обойти ограничения применимости геодезического метода изображений в лоренцевом пространстве AdS с дефицитом угла. Наш рецепт позволяет вычислять эти величины на нестационарных фонах с дефектами и легко обощается на другие многосвязные пространства с локальной AdS-метрикой. А именно:

- геодезическая двухточечная корреляционная функция продожается на случай, когда точки разделены времениподобным интервалом, путем перехода к отраженным геодезическим во времениподобной области; подходящая причинная структура лоренцевых корреляторов восстанавливается "руками" в форме дополнительных множителей специального вида;

- наше предписание можно обобщить на другие физически интересные пространства, получаемые из $\mathrm{AdS}_{3}$ при топологическом отождествлении, такие как $\mathrm{AdS}_{3}$ с несколькими частицами или пространство с черной дырой BTZ и его деформации; наш метод также можно использовать для вычисления голографической энтропии запутанности и изучения ее временной зависимости на нестационарных фоновых пространствах.

6) Похожий результат для голографической энтропии запутанности был получен из конформной теории поля в работе [13] в контексте специального локального квенча на границе. 


\section{Перенормировки отображенных геодезических в пространстве AdS с частицами}

Рассмотрим кратко схему перенормировок, использованную в работе [33] для геодезических на конусе, которая требуется для самосогласованности метода изображений.

Перенормируем длины геодезических, достигающих границы, с помощью вычитания расходящейся части. Эта перенормировка должна быть универсальной для любой геодезической и должна удовлетворять изометрии (2.5). Это приводит к тому, что в длинах отображенных геодезических появляется дополнительный численный множитель по сравнению с длиной базовой геодезической. Следовательно, в то время как вклад от последней в корреляционную функцию определяет коррелятор $G_{\Delta, 0}$, соответствующий пустому пространству AdS, вклады от отображенных геодезических определяются как

$$
\begin{aligned}
& G_{\Delta, \operatorname{ren}, n}\left(\varphi_{a, n}^{*}, t_{a, n}^{*} ; \varphi_{b}, t_{b}\right) \equiv G_{\Delta, 0}\left(\varphi_{a, n}^{*}, t_{a, n}^{*} ; \varphi_{b}, t_{b}\right) Z_{n}\left(\varphi_{a, n}^{*}, t_{a, n}^{*} ; \varphi_{b}, t_{b}\right) \\
& G_{\Delta, \operatorname{ren}, n}\left(\varphi_{a, n}^{\#}, t_{a, n}^{\#} ; \varphi_{b}, t_{b}\right) \equiv G_{\Delta, 0}\left(\varphi_{a, n}^{\#}, t_{a, n}^{\#} ; \varphi_{b}, t_{b}\right) \bar{Z}_{n}\left(\varphi_{a, n}^{\#}, t_{a, n}^{\#} ; \varphi_{b}, t_{b}\right) .
\end{aligned}
$$

Перенормировочные множители $Z_{n}$ являются комбинацией из перенормировочных коэффициентов, которые возникают после действия изометрии:

$$
\begin{aligned}
& Z_{n}\left(t_{a, n}^{\#}, \varphi_{a, n}^{\#} ; t_{b}, \varphi_{b}\right)=C_{a_{\# n}^{\#}}^{-1 / 2}=C_{a \#(n-1)}^{-1 / 2} C_{b^{*}}^{-1 / 2}=\cdots=C_{b^{* n}}^{-1 / 2}, \\
& \bar{Z}_{n}\left(t_{a, n}^{*}, \varphi_{a, n}^{*} ; t_{b}, \varphi_{b}\right)=C_{a^{*}}^{-1 / 2}=C_{a^{*(n-1)}}^{-1 / 2} C_{b^{\#}}^{-1 / 2}=\cdots=C_{b^{\# n}}^{-1 / 2},
\end{aligned}
$$

где коэффициенты определены как

$$
\begin{aligned}
C_{a \# n} & =\left(\mathcal{B}_{\xi}(-n \alpha) \cos \varphi_{a}+\sin t_{a}\left(1+2 \operatorname{sh}^{2} \xi \sin ^{2} \frac{n \alpha}{2}\right)\right)^{2}+\cos ^{2} t_{a} \\
C_{b^{* n}} & =\left(\mathcal{B}_{\xi}(n \alpha) \cos \varphi_{b}+\sin t_{b}\left(1+2 \operatorname{sh}^{2} \xi \sin ^{2} \frac{n \alpha}{2}\right)\right)^{2}+\cos ^{2} t_{b}
\end{aligned}
$$

В случае статической частицы (при $\xi=0$ ) перенормировочные множители равны единице.

Вклады от отображенных геодезических в коррелятор (П.1), таким образом, удовлетворяют свойству инвариантности относительно изометрии:

$$
\begin{aligned}
G_{\Delta, \operatorname{ren}, n}\left(\varphi_{a, n}^{*}, t_{a, n}^{*} ; \varphi_{b}, t_{b}\right) & =G_{\Delta, \operatorname{ren}, n}\left(\varphi_{a, n-1}^{*}, t_{a, n-1}^{*} ; \varphi_{b, 1}^{\#}, t_{b, 1}^{\#}\right)= \\
& =G_{\Delta, \operatorname{ren}, n}\left(\varphi_{a}, t_{a} ; \varphi_{b, n}^{\#}, t_{b, n}^{\#}\right), \\
G_{\Delta, \operatorname{ren}, n}\left(\varphi_{a, n}^{\#}, t_{a, n}^{\#} ; \varphi_{b}, t_{b}\right) & =G_{\Delta, \operatorname{ren}, n}\left(\varphi_{a, n-1}^{\#}, t_{a, n-1}^{\#} ; \varphi_{b, 1}^{*}, t_{b, 1}^{*}\right)= \\
& =G_{\Delta, \operatorname{ren}, n}\left(\varphi_{a}, t_{a} ; \varphi_{b, n}^{*}, t_{b, n}^{*}\right) .
\end{aligned}
$$

Благодарности. Авторы искренне благодарят Дмитрия Агеева за полезные обсуждения. 


\section{Список литературы}

[1] J. M. Maldacena, Adv. Theor. Math. Phys., 2:2 (1998), 231-252, arXiv: hep-th/9711200.

[2] S. S. Gubser, I. R. Klebanov, A. M. Polyakov, Phys. Lett B., 428:1-2 (1998), 105-114, arXiv: hep-th/9802109.

[3] E. Witten, Adv. Theor. Math. Phys., 2:2 (1998), 253-291, arXiv: hep-th/9802150.

[4] O. Aharony, S. S. Gubser, J. M. Maldacena, H. Ooguri, Y. Oz, Phys. Rept., 323:3-4 (2000), 183-386, arXiv: hep-th/9905111.

[5] J. Casalderrey-Solana, H. Liu, D. Mateos, K. Rajagopal, U. A. Wiedemann, Gauge/string duality, hot QCD and heavy ion collisions, arXiv: 1101.0618.

[6] И. Я. Арефьева, УФН, 184:6 (2014), 569-598.

[7] O. DeWolfe, S. S. Gubser, C. Rosen, D. Teaney, Prog. Part. Nucl. Phys., 75 (2014), 86-132, arXiv: 1304.7794.

[8] S. A. Hartnoll, C. P. Herzog, G. T. Horowitz, JHEP, 12 (2008), 015, 42 pp., arXiv: 0810.1563.

[9] S. Sachdev, "Condensed matter and AdS/CFT", From Gravity to Thermal Gauge Theories. The AdS/CFT Correspondence (Adamas, Milos Island, Greece, September 21-26, 2009), Lecture Notes in Physics, 828, ed. E. Papantonopoulos, Springer, Berlin, 2011, 273-311, arXiv: 1002.2947.

[10] V. Balasubramanian, A. Bernamonti, J. de Boer, N. Copland, B. Craps, E. Keski-Vakkuri, B. Müller, A. Schäfer, M. Shigemori, W. Staessens, Phys. Rev. D, 84:2 (2011), 026010, 31 pp., arXiv: 1103.2683.

[11] J. Aparício, E. López, JHEP, 12 (2011), 082, 34 pp., arXiv: 1109.3571.

[12] V. Keränen, E. Keski-Vakkuri, L. Thorlacius, Phys. Rev. D, 85:2 (2012), 026005, 14 pp., arXiv: 1110.5035 .

[13] C. T. Asplund, A. Bernamonti, F. Galli, T. Hartman, JHEP, 02 (2015), 171, 23 pp., arXiv: 1410.1392.

[14] E. Caceres, A. Kundu, JHEP, 09 (2012), 055, 44 pp., arXiv: 1205.2354.

[15] V. Balasubramanian, A. Bernamonti, B. Craps, V. Keränen, E. Keski-Vakkuri, B. Müller, L. Thorlacius, J. Vanhoof, JHEP, 04 (2013), 069, 50 pp., arXiv: 1212.6066.

[16] I. Aref'eva, A. Bagrov, A. S. Koshelev, JHEP, 07 (2013), 170, 15 pp., arXiv: 1305.3267.

[17] И. Я. Арефьева, ТМФ, 184:3 (2015), 398-417, arXiv: 1503.02185; I. Y. Aref'eva, AIP Conf. Proc., 1701 (2016), 090001, 5 pp.

[18] D. T. Son, A. O. Starinets, JHEP, 09 (2002), 042, 24 pp., arXiv: hep-th/0205051.

[19] S. Ryu, T. Takayanagi, Phys. Rev. Lett., 96:18 (2006), 181602, 4 pp., arXiv: hep-th/0603001.

[20] R. Callan, J. Y. He, M. Headrick, JHEP, 06 (2012), 081, 37 pp., arXiv: 1204.2309.

[21] J. Abajo-Arrastia, J. Aparicio, E. Lopez, JHEP, 11 (2010), 149, 27 pp., arXiv: 1006.4090.

[22] V. Balasubramanian, B. D. Chowdhury, B. Czech, J. de Boer, JHEP, 01 (2015), 048, 22 pp., arXiv: 1406.5859 .

[23] J. Maldacena, S. H. Shenker, D. Stanford, A bound on chaos, arXiv: 1503.01409.

[24] A. Maloney, E. Witten, JHEP, 02 (2010), 029, 58 pp., arXiv: 0712.0155.

[25] A. L. Fitzpatrick, J. Kaplan, M. T. Walters, JHEP, 08 (2014), 145, 65 pp., arXiv: 1403.6829.

[26] K. B. Alkalaev, V. A. Belavin, Nucl. Phys. B, 904 (2016), 367-385, arXiv: 1510.06685.

[27] N. Benjamin, E. Dyer, A. L. Fitzpatrick, A. Maloney, E. Perlmutter, Small black holes and near-extremal CFTs, arXiv: 1603.08524.

[28] V. Balasubramanian, S.F. Ross, Phys. Rev. D, 61:4 (2000), 044007, 12 pp., arXiv: hep-th/9906226.

[29] T. Banks, M.R. Douglas, G. T. Horowitz, E. J. Martinec, AdS dynamics from conformal field theory, arXiv: hep-th/9808016.

[30] V. E. Hubeny, M. Rangamani, Adv. High Energy Phys., 2010 (2010), 297916, 84 pp., arXiv: 1006.3675. 
[31] T. Albash, C. V. Johnson, New J. Phys., 13 (2011), 045017, 27 pp., arXiv: 1008.3027.

[32] I. Arefeva, A. Bagrov, P. Säterskog, K. Schalm, Phys. Rev. D, 94:4 (2016), 044059, 18 pp., arXiv: 1508.04440.

[33] Д. С. Агеев, И. Я. Арефьева, М. Д. Тихановская, ТМФ, 188:1 (2016), 85-120, arXiv: 1512.03362 .

[34] D. S. Ageev, I. Ya. Aref'eva, Holographic dual to conical defects: II. Colliding ultrarelativistic particles, arXiv: 1512.03363.

[35] И. Я. Арефьева, А. А. Багров, ТМФ, 182:1 (2015), 3-27.

[36] S. Deser, R. Jackiw, G. 't Hooft, Ann. Phys., 152:1 (1984), 220-235.

[37] S. Deser, R. Jackiw, Ann. Phys., 153:2 (1984), 405-416.

[38] S. Deser, R. Jackiw, Commun. Math. Phys., 118:3 (1988), 495-509.

[39] G. 't Hooft, Class. Quantum Grav., 13:5 (1996), 1023-1039, arXiv: gr-qc/9601014.

[40] I. Ya. Aref'eva, M. A. Khramtsov, JHEP, 04 (2016), 121, 22 pp., arXiv: 1601.02008.

[41] M. O. Katanaev, I. V. Volovich, Ann. Phys., 216:1 (1992), 1-28.

[42] C. A. B. Bayona, C. N. Ferreira, V. J. Vasquez Otoya, Class. Quantum Grav., 28:1 (2011), 015011, 12 pp., arXiv: 1003.5396.

[43] A. Hamilton, D. Kabat, G. Lifschytz, D. A. Lowe, Phys. Rev. D, 74:6 (2006), 066009, 15 pp., arXiv: hep-th/0606141.

[44] K. Osterwalder, R. Schrader, Commun. Math. Phys., 31:2 (1973), 83-12; 42:3 (1975), 281-305.

[45] M. Lüscher, G. Mack, Commun. Math. Phys., 41:3 (1975), 203-234.

Поступила в редакцию 17.05.2016 